\title{
Multi-criteria Optimization for Parametrizing Excess Gibbs Energy Models
}

\author{
Esther Forte ${ }^{\mathrm{a}, 1}$, Aditya Kulkarni ${ }^{\mathrm{a}}$, Jakob Burger ${ }^{\mathrm{b}, *}$, Michael Bortz $^{\mathrm{c}}$, \\ Karl-Heinz Küfer ${ }^{c}$, Hans Hasse ${ }^{a}$ \\ ${ }^{a}$ Laboratory of Engineering Thermodynamics (LTD), Technische Universität \\ Kaiserslautern, 67663 Kaiserslautern, Germany \\ ${ }^{b}$ Laboratory of Chemical Process Engineering, Campus Straubing for Biotechnology and \\ Sustainability, Technical University of Munich, 94315 Straubing, Germany \\ ${ }^{c}$ Fraunhofer Institute for Industrial Mathematics (ITWM), Fraunhofer-Platz 1, 67663 \\ Kaiserslautern, Germany
}

\begin{abstract}
Thermodynamic models contain parameters which are adjusted to experimental data. Usually, optimal descriptions of different data sets require different parameters. Multi-criteria optimization (MCO) is an appropriate way to obtain a compromise. This is demonstrated here for Gibbs excess energy $\left(G^{\mathrm{E}}\right)$ models. As an example, the NRTL model is applied to the three binary systems (containing water, 2-propanol, and 1-pentanol). For each system, different objectives are considered (description of vapor-liquid equilibrium, liquid-liquid equilibrium, and excess enthalpies). The resulting MCO problems are solved using an adaptive numerical algorithm. It yields the Pareto front, which gives a comprehensive overview of how well the given model can describe the given conflicting data. From the Pareto front, a solution that is
\end{abstract}

\footnotetext{
${ }^{*}$ Corresponding Author

Email address: burger@tum.de (Jakob Burger)

${ }^{1}$ Present Address: Evonik Technology \& Infrastructure GmbH, Rodenbacher Chaussee 4, 63457 Hanau-Wolfgang, Germany
} 
particularly favorable for a given application can be selected in an instructed way. The examples from the present work demonstrate the benefits of the MCO approach for parametrizing $G^{\mathrm{E}}$-models.

Keywords: Pareto optimization, Multi-criteria optimization, Parameter estimation, Excess free energy models, NRTL

\section{Introduction}

Thermodynamic models are essential for process design. In these models, the non-ideality of liquid mixtures is often described with models of the excess Gibbs energy $G^{\mathrm{E}}$. Two of the most widely used $G^{\mathrm{E}}$-models are NRTL [1] and UNIQUAC [2]. Their derivation is based on simple lattice models of the liquid, in which, however, binary parameters are adjusted to experimental data. The quality of the results crucially depends on the model parametrization and in particular on the choice of data that is used for this. These data can differ in various aspects: the type of data (e.g., vapor-liquid equilibrium (VLE), liquid-liquid equilibrium (LLE), excess enthalpy $h^{\mathrm{E}}$ ), the conditions for which data are available (e.g., temperature, pressure, composition), and the source of the data. Individual data sets can often be described well with $G^{\mathrm{E}}$-models after the adjustment of the parameters to the selected data. But it is also well-known that the simultaneous description of different data sets often causes problems. These problems can be related to inconsistencies in the experimental data, but they can also result from the fact that the model cannot describe the reality perfectly. A well-known example is that the simultaneous description of vapor-liquid equilibria (VLE) and liquid-liquid equilibria (LLE) with $G^{\mathrm{E}}$-models often yields only poor results [3-7]. Also 
difficulties in describing both excess enthalpy and phase equilibrium data have been reported [8 12]. Hence, the task of describing different data sets with a $G^{\mathrm{E}}$-model is often a multi-criteria optimization (MCO) problem with conflicting objectives.

These conflicts cannot be solved by the way the parametrization of the model is carried out, but there are different ways to tackle them, of which some are more appropriate than others. The common approach to include different data sets in a $G^{\mathrm{E}}$-model parameter estimation is lumping the corresponding objectives together in a single objective function, which is then minimized. Many schemes have been proposed for solving that single-objective optimization problem, e.g. see references [13-17]. Usually, weights are assigned to the different data sets in the objective function. The choice of these weights has an important influence on the result of the optimization and the performance of the model. The result of the single criteria optimization of a $G^{\mathrm{E}}$-model is a single parameter set.

Optimization problems with conflicting goals exist in many fields. In engineering and economy, they are rather the rule than the exception. They should be tackled by multi-criteria optimization, which is also known as Pareto optimization, after the Italian economist Pareto [20], who introduced the method in 1906. An MCO problem is an optimization problem which is described by a set of conflicting objective functions. A Pareto-optimal solution of such a problem is one for which an improvement in any of the objectives is not possible without a deterioration in at least one other objective. The set comprising all these solutions is the Pareto set, which is also referred to as the Pareto front of the MCO problem. 
The use of MCO has received considerable attention in recent years in different areas of chemical engineering such as process design, operation and control [21 25]. MCO is only briefly introduced here, the references [21 25] give more comprehensive introductions with a chemical engineering background. For a mathematical discussion the reader is referred to [26]. There are many methods for solving MCO problems. A common strategy is based on solving a series of single-criteria optimization problems (SCO) to obtain the Pareto front. In following this route, a question is how to set up the SCO problems in an efficient manner. The classical method consists of reformulating the original MCO problem through a weighting scalarization approach, where the objectives are combined using weighting factors, which need to be chosen in advance. Different weighting approaches have been explored [27], of which the standard weighted sum is an example. Alternative MCO methods that use a series of SCO problems comprise the so-called $\epsilon$-constraint method [26], and the goal programming method [27]. MCO problems can also be solved by using stochastic methods such as genetic algorithms, swarm approaches or adaptations of simulated annealing [27 29]. In contrast to deterministic methods, stochastic methods do not require gradient information, but they are often computationally expensive. All these methods, both deterministic and stochastic, are numerical, so that only approximations of the Pareto front are obtained. Some of the methods do, however, yield information on the accuracy of the approximation and, in some cases, it is also possible to specify the desired accuracy.

Pareto fronts can have different shapes. They are often convex, but may also contain concave parts. Some of the methods mentioned above are only 
suited for determining convex regions of the Pareto front. This is the case for the weighted sum scalarization. It resembles the traditional approach of tackling optimization problems with different goals, in which the objective functions are lumped together and SCO is used. This approach yields only a single point on the Pareto front, and it is well-known that it cannot provide solutions in non-convex regions of the Pareto front 30 32. Usually, in an optimization with this approach not only one set of weights is studied but also some alternatives. This yields a few points on convex parts of the Pareto front. From the resulting small set of optimal solutions, one solution is finally selected. MCO is different from such a mere variation of weights in the sense that it systematically yields the entire Pareto front, including the non-convex regions.

The present study complements previous work of our group, in which MCO was used for the parametrization of molecular force fields of fluids 3335] as well for the parametrization of equations of state [36]. In most of these works, an adaptive numerical scheme based on a sandwiching and a hyperboxing algorithm [37 39] was used for determining the Pareto front with a given accuracy. That algorithm is also suited for the parametrization of $G^{\mathrm{E}}$-models by MCO and was therefore used in the present work. The NRTL model [1] is used here as an example. The MCO method which is presented here can be applied directly to other $G^{\mathrm{E}}$-models like UNIQUAC [2].

To the best of the authors' knowledge, there is only one previous example on the application of MCO to the parametrization of $G^{\mathrm{E}}$-models [40]. The authors of this conference paper briefly describe the application of MCO for parametrizing the NRTL model using an evolutionary algorithm which was 
combined with the weighted-sum scalarization approach. In the present work, the application of MCO to $G^{\mathrm{E}}$-model parametrization is addressed more systematically using an adaptive algorithm to compute the Pareto front in a computationally-efficient manner, rendering also Pareto-optimal solutions in non-convex regions of the Pareto front. Furthermore, in contrast to [40], the quality of the numerical approximations of the Pareto front can be specified in the present algorithm. Three binary mixtures of the components water, 2-propanol and 1-pentanol were considered here as examples. The studied objectives were the description of the vapor-liquid equilibrium, the liquid-liquid equilibrium and the excess enthalpies. As MCO yields an overview of what can be achieved with a given model regarding the representation of a certain set of data, the results are particularly useful for comparing different models or different variants of the same model. This enables us to discuss some longstanding issues regarding $G^{\mathrm{E}}$-models, such as using temperature-independent vs. temperature-dependent parameters, using two or three adjustable binary parameters, and simultaneously describing different types of data.

This paper is organized as follows: the adaptive algorithm that was used in this work to compute Pareto fronts is introduced in Section 2, followed by details regarding the objective functions and the application to the NRTL model. In Section 3, an overview of the MCO problems studied in the present work, i.e. the objectives considered for each system, is given. In Section 4 the Pareto fronts are presented and discussed. First, MCO problems with two adjustable binary parameters are considered followed by a discussion of different model variants with varying numbers of parameters. Finally, an application of the results to ternary systems is discussed. Conclusions are 
drawn in Section 5 .

\section{Methodology}

\subsection{General optimization task}

The general optimization task of the present study can be formulated as follows: let there be a number of experimental data sets which shall be described with a given $G^{\mathrm{E}}$-model, and let there be a conflict between the objectives of describing the individual data sets. The task is to find all Pareto-optimal solutions of this problem. Each of these solutions is characterized by the value of the different objective functions, as well as the value of the corresponding parameters of the $G^{\mathrm{E}}$-model, i.e. by a point in the objective space and the corresponding point in the parameter space. The Pareto front represents the set of optimal solutions in the objective space and has a picture in the parameter space. For a given application, e.g. a process design, a particularly attractive solution can be selected from the Pareto front in the objective space and the corresponding parameters can be found in the parameter space. This choice is an instructed one, as the entire Pareto front is known.

\subsection{Algorithm for finding an approximation of the Pareto front}

An adaptive sampling algorithm was used here to compute the Pareto front in an efficient manner, following previous works [37 39]. The same method has furthermore been applied successfully in previous studies for solving MCO tasks in product and process design [39, 41 46], optimal experimental design [47], parameter estimation of molecular simulations [35] and 
parameter estimation of equations of state [36]. It is an algorithm 37 39] that calculates a minimal number of Pareto-optimal solutions and the linear interpolation between them, which approximates the Pareto front within a predefined quality. The algorithm is a hybrid method that combines a sandwiching algorithm to sample convex regions, and a hyperboxing scheme to sample non-convex regions of the Pareto front. In both cases a scalarization of the objectives is performed, such that each Pareto-optimal solution is computed by a deterministic single-objective optimization algorithm. Within the sandwiching scheme a weighted-sum scalarization is employed [26], whereas within the hyperboxing approach a Pascoletti-Serafini scalarization [48] is used. In these scalarizations, the previously calculated solutions are taken into account making the algorithm adaptive and computationally efficient. The approach has already been described in detail in previous works [3739], to which the reader is referred to for more details. The implementation details are essentially the same as described in [36].

\subsection{Definition of the conflicting objectives}

Different competing objective functions are used in the present work, which quantify deviations between the model and experimental data for various types of phase equilibria or properties. Average absolute deviations in the following quantities were considered: mole fractions in vapor-liquid equilibria $x_{\mathrm{VLE}}$, mole fractions in liquid-liquid equilibria $x_{\mathrm{LLE}}$, and excess enthalpies $h^{\mathrm{E}}$. The objective functions are defined as follows:

$$
\delta x_{\mathrm{VLE}}(\boldsymbol{\Omega})=\frac{1}{N_{\mathrm{VLE}}} \sum_{i=1}^{N_{\mathrm{VLE}}} \sum_{l=1}^{N_{\mathrm{P}}}\left|x_{1}^{l, \text { calc }}\left(T_{i}, p_{i} ; \boldsymbol{\Omega}\right)-x_{1}^{l, \exp }\left(T_{i}, p_{i}\right)\right|,
$$




$$
\delta x_{\mathrm{LLE}}(\boldsymbol{\Omega})=\frac{1}{N_{\mathrm{LLE}}} \sum_{i=1}^{N_{\mathrm{LLE}}} \sum_{l=1}^{N_{\mathrm{P}}}\left|x_{1}^{l, \text { calc }}\left(T_{i}, p_{i} ; \boldsymbol{\Omega}\right)-x_{1}^{l, \exp }\left(T_{i}, p_{i}\right)\right|,
$$

and

$$
\delta h^{\mathrm{E}}(\boldsymbol{\Omega})=\frac{1}{N_{h^{\mathrm{E}}}} \frac{1}{h_{\max }^{\mathrm{E}, \exp }} \sum_{i=1}^{N_{\mathrm{hE}}}\left|h^{\mathrm{E}, \mathrm{calc}}\left(T_{i}, p_{i}, x_{i} ; \boldsymbol{\Omega}\right)-h^{\mathrm{E}, \exp }\left(T_{i}, p_{i}, x_{i}\right)\right|,
$$

where $i$ indicates the experimental data point, and $l$ indicates the equilibrium phase $l$. $N_{\mathrm{VLE}}, N_{\mathrm{LLE}}$, and $N_{h^{\mathrm{E}}}$ are the number of experimental data points of each type that are used, $T_{i}$ is the absolute temperature, $p_{i}$ is the pressure, $\Omega$ is the set of NRTL parameters, $N_{\mathrm{P}}$ is the number of phases (i.e., deviations are computed for both phases in equilibrium), and $h_{\max }^{\mathrm{E}, \mathrm{exp}}$ is the maximum absolute value of the experimental excess enthalpy in the data set. The pressure dependence of the state variables considered in Eqs. (2) and (3) is weak and not accounted for in the NRTL model.

The objective functions that were used here should be considered as mere examples; as such, they were kept simple and similar. In principle, any objective function can be used in the MCO method.

\subsection{NRTL model}

The molar excess Gibbs free energy for a binary liquid mixture of components 1 and 2 is expressed in the NRTL model as [1]:

$$
\frac{g^{\mathrm{E}}}{R T}=x_{1} x_{2}\left(\frac{\tau_{21} G_{21}}{x_{1}+x_{2} G_{21}}+\frac{\tau_{12} G_{12}}{x_{2}+x_{1} G_{12}}\right),
$$

where $R$ is the ideal gas constant, and $G_{i j}=\exp \left(-\alpha \tau_{i j}\right)$. The terms $\tau_{i j}$ and $\tau_{j i}$ depend on the temperature. In the present work, two forms to cor- 
relate this temperature dependence are studied. If not stated otherwise, Equation (5) is used:

$$
\tau_{i j}=\frac{b_{i j}}{T} .
$$

Here, $b_{i j}$ and $b_{j i}$ are two adjustable parameters. As an alternative, we have also studied a form in which a linear temperature dependence is assumed, and which has four adjustable parameters:

$$
\tau_{i j}=a_{i j}+\frac{b_{i j}}{T} .
$$

The non-randomness parameter $\alpha$ of the NRTL model is not always adjusted. If it is fixed, usually values in the range from 0.2 to 0.5 are chosen [49]. We compare variants with fixed $\alpha$ and adjusted $\alpha$ in the present work.

\subsection{Calculation of thermodynamic properties}

The vapor-liquid equilibrium was calculated from:

$$
p_{i}^{\mathrm{s}} x_{i} \gamma_{i}=p y_{i},
$$

where $\gamma_{i}$ is the activity coefficient of component $i$ in the liquid phase, $x_{i}$ is its mole fraction in the liquid phase, $y_{i}$ is its mole fraction in the vapor phase and $p$ is the pressure. The vapor pressures $p_{i}^{\mathrm{s}}$ of the pure components were calculated using an extended form of the Antoine equation [50], as specified in the Supplementary Material.

The liquid-liquid equilibrium between two liquid phases I and II was calculated from

$$
x_{i}^{\mathrm{I}} \gamma_{i}^{\mathrm{I}}=x_{i}^{\mathrm{II}} \gamma_{i}^{\mathrm{II}} .
$$


The excess enthalpy was obtained from

$$
\frac{h^{\mathrm{E}}}{\mathrm{R} T^{2}}=-\left[\frac{\partial\left(g^{\mathrm{E}} / \mathrm{R} T\right)}{\partial T}\right]
$$

\section{Overview of the studied MCO problems}

The MCO problems that were studied in the present work are case studies, in which the task was to find optimal parametrizations of the NRTL model for representing experimental data for binary systems. The three binary mixtures that can be obtained from water, 2-propanol, and 1-pentanol were taken as examples. This choice is not motivated by a special application, but rather by the desire to limit the number of substances in the study and to find sufficiently interesting data sets of different nature for all binary systems.

Figure 1 gives an overview of the $\mathrm{MCO}$ problems that are solved in the present work. For each binary system, two sets of thermodynamic data of different nature were used for defining objective functions according to Equations (1) to (3). For the system water +1 -pentanol the task was to simultaneously describe VLE and LLE. For the system 2-propanol + 1-pentanol as well as for the system water + 2-propanol, the task was to simultaneously describe VLE and $h^{\mathrm{E}}$. The sources of the data that were used here are specified in Table 1. These are not the only experimental data available for the systems that were studied here. As our work focuses on the MCO method and the data serve basically only for illustrating the procedure, just a single data set for each property and system was considered to keep the presentation concise and focused on the main issue, i.e. MCO.

An extension of the study to MCO problems that also include data for 


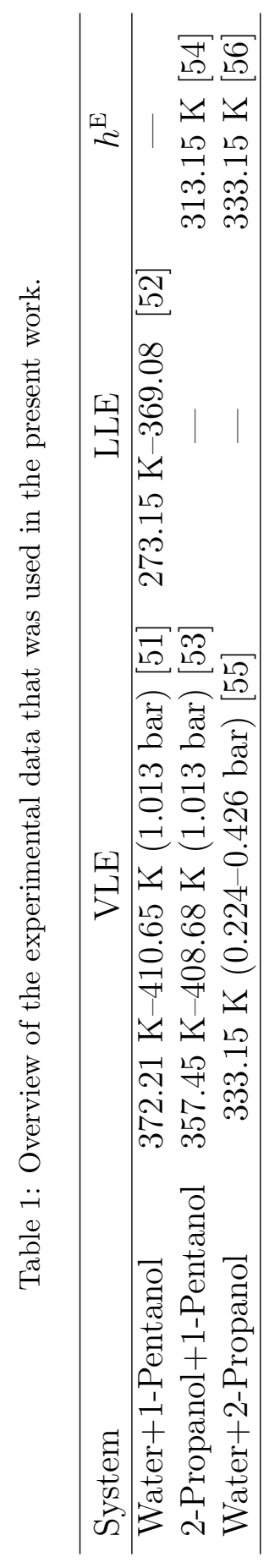


the ternary mixture water +2 -propanol +1 -pentanol would be possible, but was not attempted here, as ternary data are usually not included in the estimation of binary interaction parameters. Only for completeness, some predictions of ternary data with models obtained from optimizations to the binary data are shown.

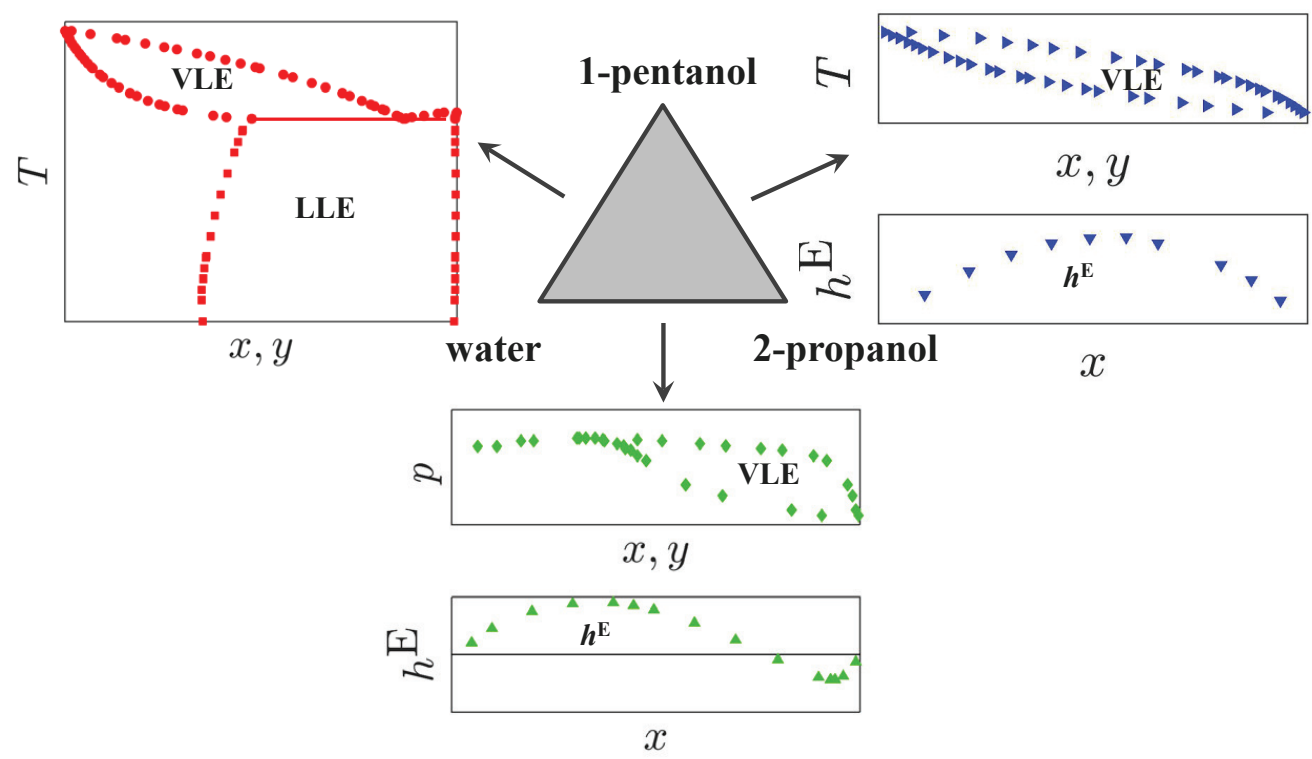

Figure 1: Overview of the MCO problems that are solved in the present study. The symbols represent experimental data which are only shown qualitatively for illustration. The actual data that were used are specified in Table 1

\section{Results and discussion}

\subsection{Pareto fronts for a fixed value of the non-randomness parameter}

The present subsection reports on results of a study in which the nonrandomness parameter of the NRTL model was set to $\alpha=0.3$. Hence, there were only two adjustable parameters: $b_{i j}$ and $b_{j i}$, cf. Equation (5). Figure 2 
shows the Pareto fronts that were obtained for the three MCO problems defined in Section 3. Figure 2 contains the results for each of the studied binary systems, i.e. (from top to bottom): water +1 -pentanol, 2-propanol + 1-pentanol, and water + 2-propanol.

On the left side of Figure 2, the Pareto fronts of the three MCO problems are shown. The objective space is two-dimensional in all cases: on the $\mathrm{x}$ axis of the plots the deviation in the first objective is indicated, which is the description of the VLE $\left(\delta x_{\mathrm{VLE}}\right)$ in all cases; on the y-axis, the deviation in the second objective is indicated, which is either the description of the LLE $\left(\delta x_{\mathrm{LLE}}\right)$ or that of $h^{\mathrm{E}}\left(\delta h^{\mathrm{E}}\right)$, respectively, cf. Equations (1) to (3). In each diagram, also the two extreme compromises are displayed (by dashed lines), i.e. the optima that are obtained for the case that only one of the objectives is considered. The Pareto front is plotted only in a certain range, such that the extreme compromises can only be seen in some of the plots, whereas they lie outside the depicted range in others. Excellent descriptions of the VLE can be obtained with the NRTL model, when only one objective is considered (i.e. for extreme compromises), in all studied cases, with a lower limit of $\delta x_{\mathrm{VLE}}$ of about $1 \mathrm{~mol} \%$ for the systems water +1 -pentanol and 2propanol + 1-pentanol, and about $2 \mathrm{~mol} \%$ for water +2 -propanol. Good results can also be achieved for the second objective in the first two systems: $\delta x_{\mathrm{LLE}}=3.5 \mathrm{~mol} \%$ for water +1 -pentanol and $\delta h^{\mathrm{E}}=1 \%$ for 2-propanol + 1-pentanol, while for water +2 -propanol the best value that can be obtained for $\delta h^{\mathrm{E}}$ is about $8 \%$.

The Pareto fronts connect these extreme compromises. All Pareto fronts presented in Figure 2 are convex. Upon approaching an extreme compromise 

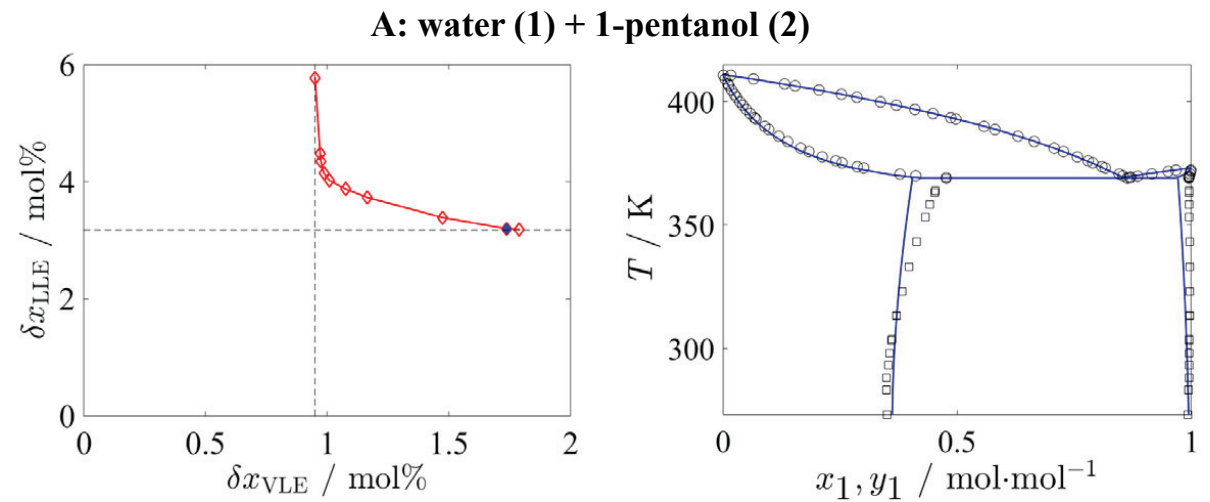

B: 2-propanol (1) + 1-pentanol (2)
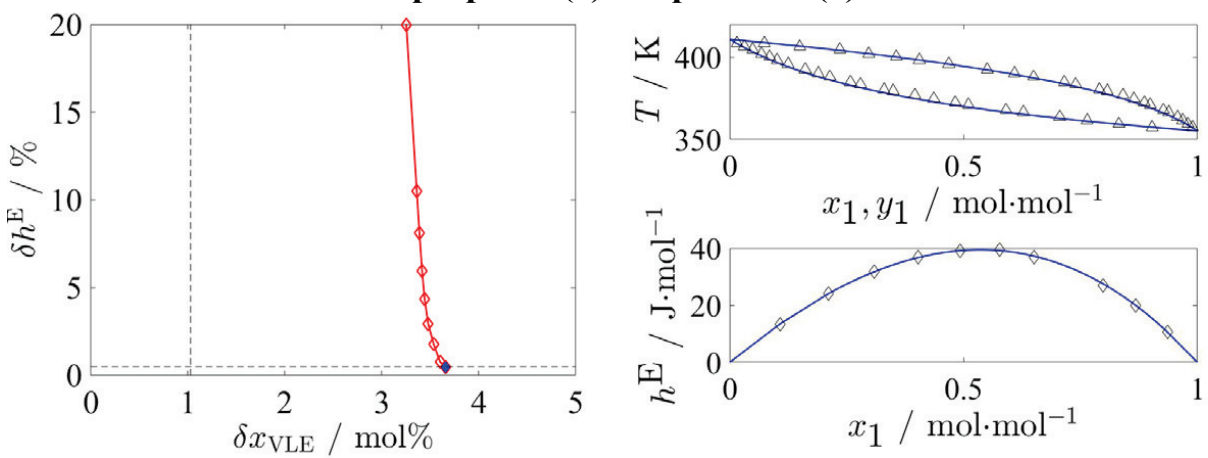

C: water (1) + 2-propanol (2)
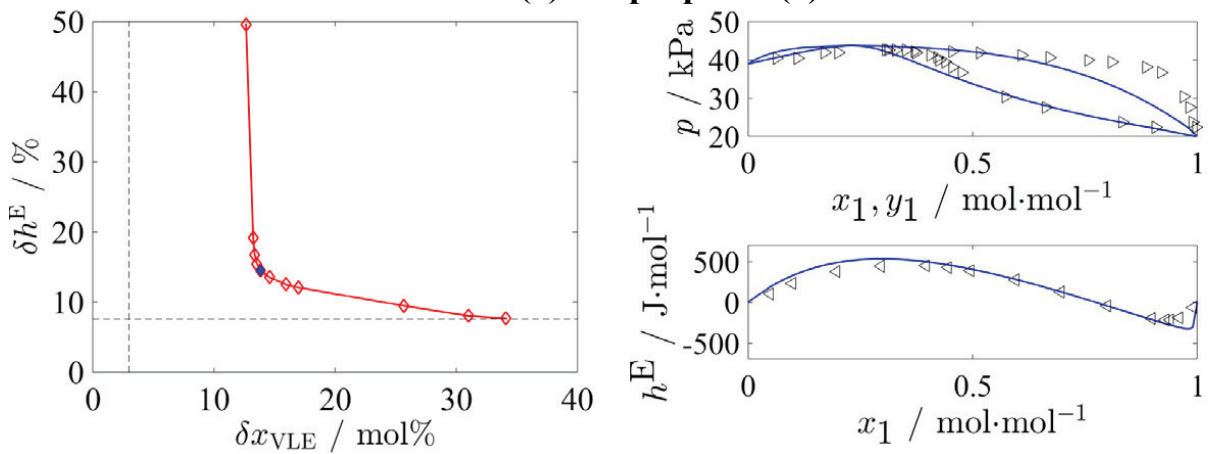

Figure 2: Results from the solution of MCO problems in which two NRTL parameters $\left(b_{i j}\right.$ and $b_{j i}$, cf. Equation (5) were fitted to different sets of experimental data, see Table 1 . $\alpha=0.3$ in all cases. Left column: Pareto fronts (symbols: Pareto-optimal solutions, lines: approximation of the Pareto front). The results for the extreme compromises are indicated as dashed lines. One model (marked in blue) was selected from each Pareto front (see text). Right column: description of the data sets that were used in the fit by the selected Pareto-optimal model (symbols: experimental data [51 56] lines: model). 
in one objective, often heavy losses in the other objective have to be accepted for small improvements in the considered objective. The part of the Pareto fronts that has the strongest curvature is often called the Pareto knee. A typical Pareto knee is observed for the system water +2 -propanol, see Panel $\mathrm{C}$ in Figure 2. When the Pareto knee is sharp, the most attractive models are typically found in the region of that knee. An example for such a choice of a model in the region of the Pareto knee is depicted in Panel $\mathrm{C}$ of Figure 2 by the blue symbol.

Each set of the NRTL model parameters maps to a distinct point in the objective space and vice versa. Thus, there is a picture of the Pareto front in the parameter space. With the model parameters corresponding to the selected point on the Pareto front in Panel C of Figure 2 (blue symbol), the quality of the fit of the underlying data was investigated in more detail. The result is shown on the right side of Figure 2, Panel C, where the diagrams for the VLE and $h^{\mathrm{E}}$ are presented. The fit of the VLE of the system water $+2-$ propanol by the selected model leaves ample room for improvement. Indeed, the corresponding Pareto front on the left side of Figure 2 reveals that much better descriptions of the VLE are possible, but only at the expense of a very important deterioration of the description of $h^{\mathrm{E}}$. This illustrates that the comprehensive overview of all relevant solutions of the MCO problem given by the Pareto front enables an instructed choice of an attractive solution.

Panels A and B of Figure 2 provide two other examples. Also the Pareto front shown in Panel A for the system water +1 -pentanol has a Pareto knee. Nevertheless, rather than selecting a solution from the Pareto knee, a solution near the extreme compromise regarding the LLE was chosen (blue 
point in Panel A of Figure 2). The reason for this choice is simply that the description of the VLE in this point is not much worse than that for solutions from the Pareto knee. The quality of the VLE fit can be assessed using the diagram on the right side of Panel A in Figure 2. However, that panel shows that, even though high emphasis was put on the description of the LLE, the fit of the LLE is still not very good. The Pareto front shows that the model simply cannot describe the LLE data well.

The third example is shown in Panel B of Figure 2, in which results for the system 2-propanol +1 -pentanol are depicted. The left side shows again the Pareto front. Here, the extreme compromise regarding $\delta h^{\mathrm{E}}$ is selected from the Pareto front for a closer inspection. The reason for this choice is that the Pareto front is so steep, that starting from the selected point, the small improvement that could be obtained in the VLE would not justify the high deterioration in the description of $h^{\mathrm{E}}$. The resulting fits of the VLE and of $h^{\mathrm{E}}$ are shown on the right side of Panel B in Figure 2. Excellent agreement is obtained for both studied properties, which is not astonishing as the system shows no strong deviations from ideality.

In the examples studied in the present subsection, the parameter space is two-dimensional (parameters $b_{i j}$ and $b_{j i}$ ). Figure 3 shows the contour plots of the deviations in both the objectives in the parameter space, which were obtained by brute force calculations. For each point of the Pareto front (in the objective space), cf. the left-hand side of Figure 2, there is a corresponding point in the parameter space. White curves depict the pictures of the Pareto fronts in the parameter space. There are six panels in Figure 3. The three columns correspond to the three different MCO problems (the three systems) 
and the two rows correspond to the two data types (the objectives) considered for each of the systems. For a given column, the curve representing the image of the Pareto front is therefore identical in both the upper and the lower plot. The parameter combination that corresponds to the extreme compromises in $\mathrm{MCO}$, are indicated in the plot by red crosses. In the case of the system water +2 -propanol, the picture of the extreme compromise giving the best VLE description lies far outside the depicted parameter ranges and it is not shown to maintain a good resolution of the interesting region. The Pareto front connects the two extreme compromises, and therefore the picture of the Pareto front in the parameter space connects the points that correspond to the optima of the SCO problems. The connecting line is highly nonlinear. The parameters of the Pareto-optimal solutions that were selected from the Pareto fronts are also indicated in Figure 3 (by cyan crosses) for completeness.

\subsection{Influence of the non-randomness parameter}

Pareto fronts provide excellent means for a fair and comprehensive comparison of different models (or different variants of the same model) regarding the description of a certain set of experimental data. This is illustrated here by comparing different variants of the NRTL model. The systems and objectives are the same as in the previous section.

Figure 4 shows Pareto fronts that were obtained for MCO problems in which different values of $\alpha$ were fixed and the remaining two NRTL parameters $\left(b_{i j}\right.$ and $\left.b_{j i}\right)$ were fitted. This is basically an extension of the results shown in Figure 2 to different values of $\alpha$. Additionally, the Pareto front of the corresponding MCO problem, in which three NRTL parameters $\left(b_{i j}, b_{j i}\right.$, 


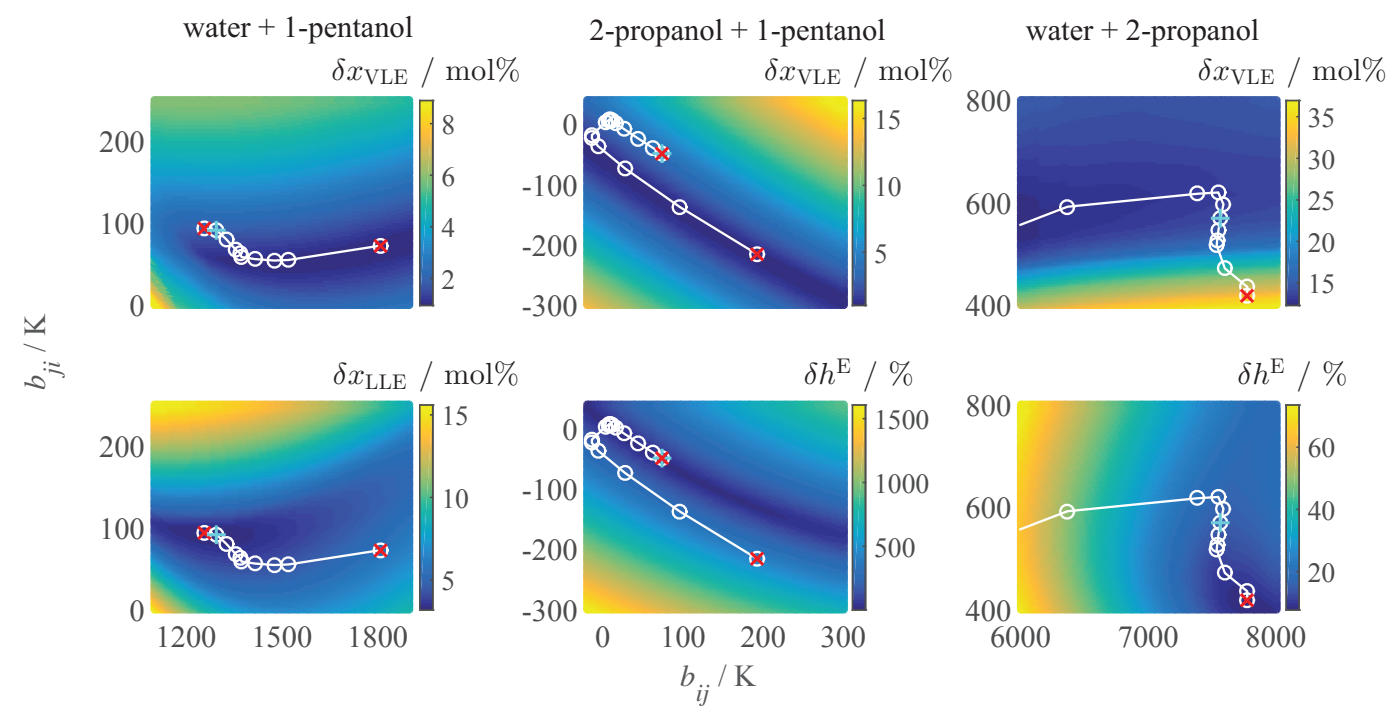

Figure 3: Contour plots of deviations in different objectives for the three studied binary systems in the parameter space for the case of the adjustment of two NRTL parameters $\left(b_{i j}\right.$ and $\left.b_{j i}\right) . \alpha=0.3$ in all cases. In the upper row, deviations for the VLE are shown, in the lower row, deviations for the second property, which was either LLE or $h^{\mathrm{E}}$. The white curve is the image of the Pareto front that was obtained for the system (cf. Figure 2 ) in the parameter space. The open circles correspond to computed points on the Pareto front; the lines are linear interpolations. The red crosses correspond to the extreme compromises and the cyan crosses to the selected Pareto-optimal solutions (cf. Figure22).

and $\alpha$ ) were fitted, is shown for each of the three studied systems. Not astonishingly, in a certain range of $\alpha$, the Pareto fronts obtained for fixed $\alpha$ touch the Pareto front for optimized $\alpha$; and the Pareto front for the MCO problem with variable $\alpha$ is the envelope of all Pareto fronts of the corresponding MCO problems with fixed $\alpha$.

For the systems water +1 -pentanol and water +2 -propanol the choice of $\alpha$ has a strong influence on the results. The results presented in Figure 4 illustrate that the best choice for $\alpha$ depends on the preferences of the user regarding the objectives. They also show that the choice of $\alpha$ may have a 

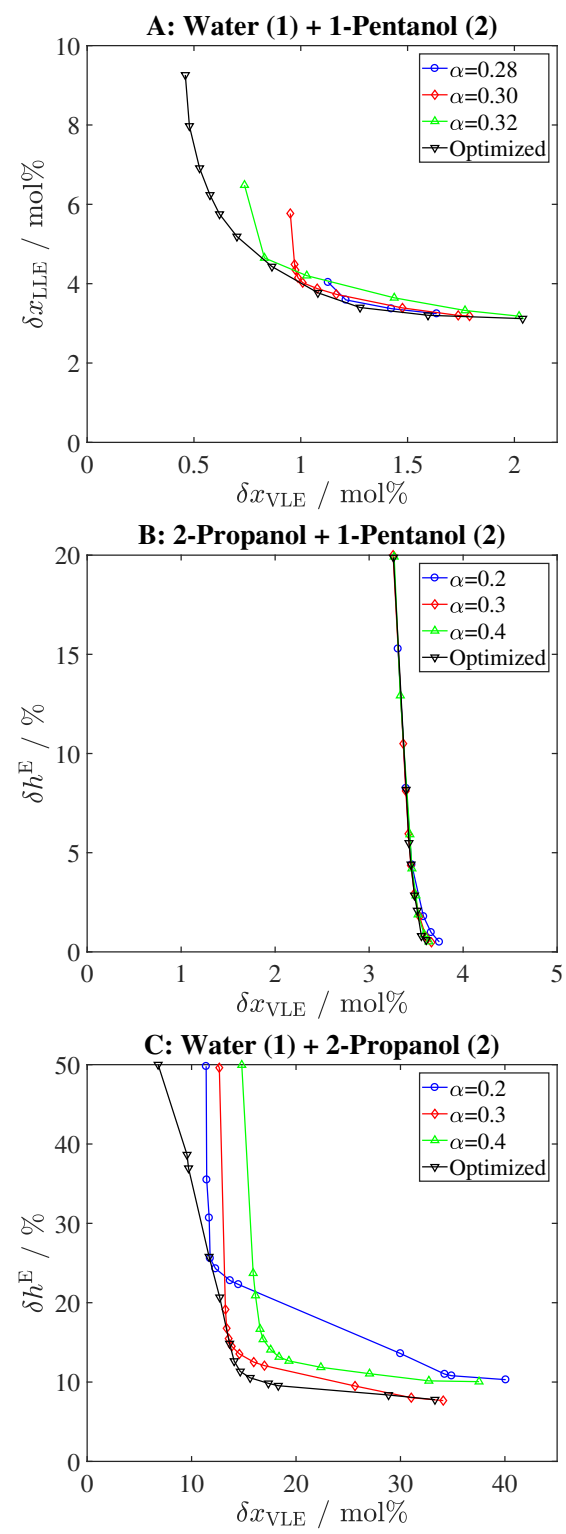

Figure 4: Pareto fronts of the solution of MCO problems in which two NRTL parameters $\left(b_{i j}\right.$ and $b_{j i}$, cf. Equation (5) were fitted to different sets of experimental data, see Table 1 . Different fixed values for $\alpha$ are used. The systems and objectives are the same as in the MCO problems in Figure 2. Additionally, the Pareto front of the corresponding MCO problem, in which three NRTL parameters $\left(b_{i j}, b_{j i}\right.$, and $\left.\alpha\right)$ were fitted, is shown. In a certain range of $\alpha$, the Pareto fronts obtained for fixed $\alpha$ touch the Pareto front for optimized $\alpha$. 
considerable influence on the position of the limits obtained in the case of the extreme compromises: this is the case for the VLE in the system water +1 -pentanol and for $h^{\mathrm{E}}$ in the system water +2 -propanol. In contrast, for the system 2-propanol +1 -pentanol, the choice of $\alpha$ has little influence on the quality of the fit.

For all three systems, it turns out that $\alpha=0.3$ is a fair choice. The benefits that can be gained from adjusting $\alpha$ besides the other two NRTL parameters are not large compared to results that are obtained for a fixed value of $\alpha=0.3$. Therefore, no plots are presented here, in which results for a selected model from the Pareto front of the three-parameter fit are compared to experimental data; they would be similar to those on the right side of Figure 2 .

\subsection{Effect of additional parameters in the temperature dependence}

In the present study, two different forms of the temperature dependence of the terms $\tau_{i j}$ and $\tau_{j i}$ were considered. The results obtained from the twoparameter version in which the parameters are $b_{i j}$ and $b_{j i}$, cf. Equation (5), have already been discussed in Section 4.1. In the present section, the four parameter version with the two additional parameters $a_{i j}$ and $a_{j i}$, cf. Equation (6), is investigated and compared to the two-parameter version. A fixed value of $\alpha=0.3$ was used in both the cases. The objectives are unchanged compared to the study described above. Only the system water +1 -pentanol is studied for brevity. In Figure 5, the Pareto fronts that were obtained with the two versions of the description of the temperature dependence of NRTL parameters are compared for this system. The Pareto fronts for the twoparameter form are the same as in Figure 2, as the objectives are the same. 
Using the four-parameter version leads to an important improvement: the results for the extreme compromises are shifted to distinctly lower deviations in both the objectives. From the Pareto fronts of the four parameter version, a solution was selected (full symbol) and the results for that solution are compared to the experimental data sets that were used for the fit in the diagram on the right side of Figure 5. This diagram can be compared directly with the corresponding one in Figure 2, Panel A, which shows the results obtained with the two-parameter version. A better simultaneous description of both the VLE and the LLE data is obtained with the four-parameter version.
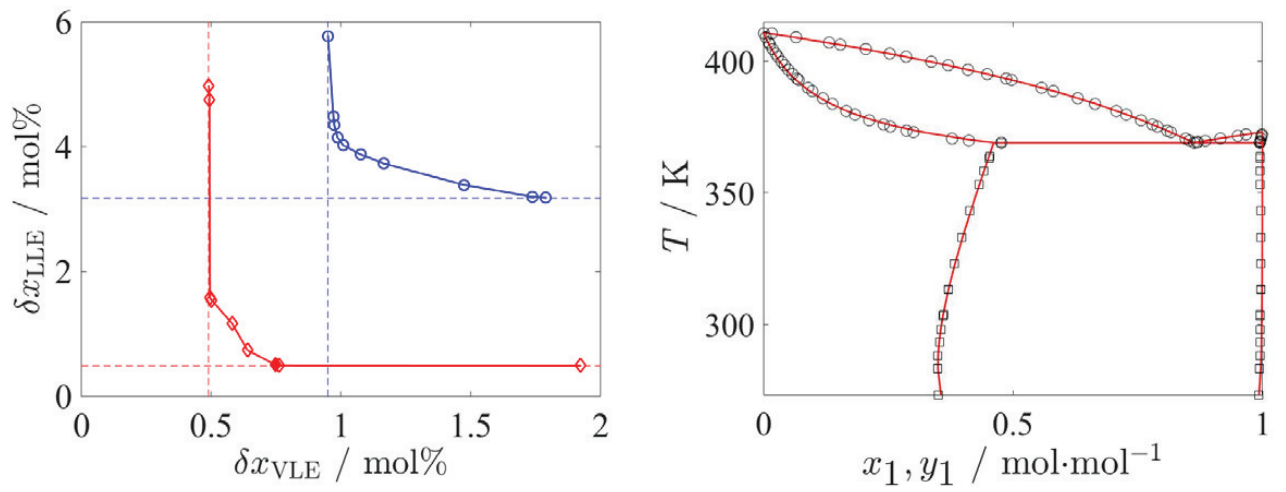

Figure 5: Results from the solution of MCO problems in which four NRTL parameters $\left(a_{i j}, a_{j i}, b_{i j}\right.$ and $b_{j i}$,cf. Equation (6) $)$ were fitted to different sets of experimental data (see Table 1) along with comparison with corresponding results from two-parameter fit $\left(b_{i j}\right.$ and $b_{j i}$, cf. Equation (5p). $\alpha=0.3$ in all cases. Left column: Pareto fronts (symbols: Pareto-optimal solutions, lines: approximation of the Pareto front). The results for the extreme compromises are indicated as dashed lines. One model (full symbol) was selected from the Pareto front. Right column: description of the data sets that were used in the fit by the selected Pareto-optimal model (symbols: experimental data, lines: model). The results shown on the right side can be compared directly with those from the selected model of the two-parameter version shown in Figure 2 . 


\subsection{Prediction of ternary phase behavior}

Having the possibility of examining the trade-offs existing in the description of different properties for each binary pair can be an advantage for the selection of parameters to model the ternary system. Additionally, the Pareto front for each binary pair provides the decision maker with a number of optimized available options. The performance of different possible models can therefore be examined, without the need of carrying out further optimizations.

We do not enter into a detailed discussion here and simply give an example of the application of the results obtained for the binary systems to a ternary system: the optimal models selected at a fixed value of $\alpha=0.3$ (see Figure 2) are used to predict LLE of the ternary mixture water +1 -pentanol +2 propanol. The results are presented in Figure 6 .
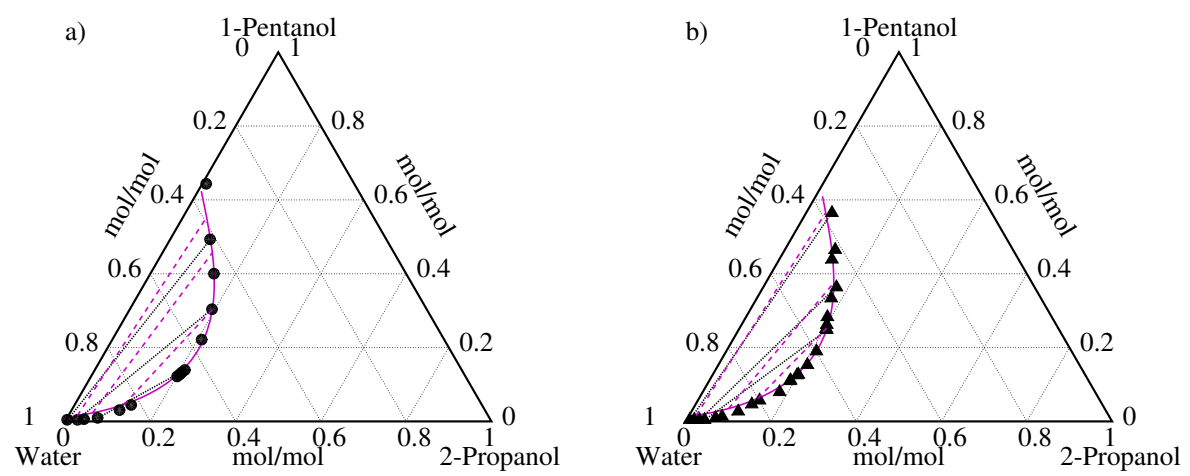

Figure 6: LLE of the system water + 2-propanol + 1-pentanol at a) $293 \mathrm{~K}$ and b) 298 K. The symbols correspond to experimental data (circles [57, triangles [58]), the solid lines correspond to the predictions with the NRTL model, the dotted lines represent experimental tie-lines and the dashed lines represent calculated tie-lines. The predictions were obtained with the parameters of three Pareto-optimal models for the three binary sub-systems indicated on the Pareto fronts in Figure 2 


\section{Conclusions}

In previous works, we have shown that MCO is an interesting tool for parameterizing molecular models and equations of state; in the present work we show that this is also true for $G^{\mathrm{E}}$-models. An efficient algorithm was used to calculate Pareto fronts for a number of $\mathrm{MCO}$ problems, in which the task was to find optimal NRTL parameters for describing different data sets. Three different binary test systems were studied. For each of these systems, two objectives were considered, which consisted in obtaining a good description of experimental data sets of different types (vapor-liquid equilibrium, liquidliquid equilibrium and excess enthalpies). As the model is not perfect, there is a conflict between the objectives: parameters that are optimal in respect to one objective lead to suboptimal solutions for the other. The Pareto front connects these two extreme cases and gives a comprehensive overview of how well the model can describe the given set of conflicting data. It is therefore an excellent mean for comparing different models or different variants of the same model. This was demonstrated here for different variants of the NRTL model. In a first case study, the two-parameter variant of the model (with fixed $\alpha$ ) was compared to the three-parameter variant (with adjustable

$\alpha$ ). In a second case study, the influence of the temperature dependence of the NRTL parameters was investigated. By comparing the Pareto fronts for the different variants, the benefits that can be drawn from introducing more parameters can be assessed.

MCO differs strongly from the traditional approach to include different objectives in the parametrization of models, which relies on lumping the objectives in a single objective function, which is then minimized. The result 
is then a single optimal solution, which, however, strongly depends on the way the lumping was carried out, in particular on the choice of weights for different objectives. Different models or model variants can thereby only be compared point-wise. MCO overcomes this problem by supplying the complete set of optimal solutions, the Pareto front, from which a solution, which is particularly attractive for a given application can be selected. The selection is instructed, as the entire set of optimal solutions is known. This is in contrast to the traditional approach, in which weights for the different objectives have to set before carrying out the optimization. By varying the weights, different points on the Pareto front can be sampled, if the front is convex. But this method fails for non-convex Pareto fronts and is usually inefficient if carried out manually. To conclude: MCO is the method of choice for parameterizing $G^{\mathrm{E}}$-models in all cases in which compromises between different objectives are necessary.

\section{Acknowledgements}

The authors acknowledge financial support from the Reinhart-Koselleck Programme (HA1993/15-1) of the German Research Foundation (DFG).

\section{Bibliography}

[1] H. Renon, J. M. Prausnitz, Local compositions in thermodynamic excess functions for liquid mixtures, AIChE J. 14 (1) (1968) 135-144.

[2] D. Abrams, J. M. Prausnitz, Statistical thermodynamics of liquid mixtures: A new expression for the excess gibbs energy of partly or completely miscible systems, AIChE J. 21 (1) (1975) 116. 
[3] G. M. Lobien, J. M. Prausnitz, Infinite-dilution activity coefficients from differential ebulliometry, Ind. Eng. Chem. Fund. 21 (2) (1982) 109-113.

[4] S. Zeck, Thermodynamics in process development in the chemical industry - importance, benefits, current state and future development, Fluid Phase Equilib. 70 (2-3) (1991) 125-140.

[5] J. D. Raal, C. J. Brouckaert, Vapour-liquid and liquid-liquid equilibria in the system methyl butenol-water, Fluid Phase Equilib. 74 (1992) 253 $-270$.

[6] L.-S. Lee, W.-C. Chen, J.-F. Huang, Experiments and correlations of phase equilibria of ethanol-ethyl acetate-water ternary mixture, J. Chem. Eng. Jpn. 29 (3) (1996) 427-438.

[7] V. Gomis, A. Font, M. D. Saquete, J. Garca-Cano, Liquidliquid, vaporliquid, and vaporliquidliquid equilibrium data for the waternbutanolcyclohexane system at atmospheric pressure: Experimental determination and correlation, J. Chem. Eng. Data 58 (12) (2013) 33203326.

[8] G. L. Nicolaides, C. A. Eckert, Optimal representation of binary liquid mixture nonidealities, Ind. Eng. Chem. Fund. 17 (4) (1978) 331-340.

[9] R. W. Hanks, R. L. Tan, J. J. Christensen, Limits on the simultaneous correlation of $g^{\mathrm{E}}$ and $h^{\mathrm{E}}$ data by the NRTL, LEMF and Wilson's equations, Thermochimica Acta 23 (1) (1978) 41 - 55. 
[10] J. R. Battler, W. M. Clark, R. L. Rowley, Excess enthalpy and liquidliquid equilibrium surfaces for the cyclohexane-2-propanol-water system from 293.15 to 323.15 k, J. Chem. Eng. Data 30 (3) (1985) 254-259.

[11] Y. Demirel, H. Gecegörmez, Simultaneous correlation of excess gibbs energy and enthalpy of mixing by the UNIQUAC Equation, Can. J. Chem. Eng 67 (3) (1989) 455-461.

[12] A. U. Burman, K. H. U. Ström, Calculation of vapor liquid equilibrium and excess enthalpy with the UNIQUAC Equation, Chem. Eng. Commun. 194 (8) (2007) 1029-1052.

[13] T. F. Anderson, D. S. Abrams, E. A. Grens, Evaluation of parameters for nonlinear thermodynamic models, AIChE J. 24 (1) (1978) 20-29.

[14] D. Salazar-Sotelo, A. Boiveaut Boudehen, H. Renon, Computer calculation of the optimal parameters of a model for the simultaneous representation of experimental binary and ternary vapor-liquid and liquid-liquid equilibrium data, Fluid Phase Equilib. 27 (1986) 383 - 403.

[15] P. Englezos, N. Kalogerakis, Applied parameter estimation for chemical engineers, CRC Press, 2000.

[16] K. A. G. Schmidt, Y. Maham, A. E. Mather, Use of the NRTL equation for simultaneous correlation of vapour-liquid equilibria and excess enthalpy, J. Therm. Anal. Calorim. 89 (1) (2007) 61-72.

[17] K. Tochigi, J. Rarey, J. Gmehling, Recommended NRTL model parameters by simultaneous correlation of VLE, infinite dilution activity 
coefficients and excess enthalpy data, J. Chem. Eng. Jpn. 42 (6) (2009) 376-380.

[18] D. Mehta, C. Grosan, A collection of challenging optimization problems in science, engineering and economics, in: 2015 IEEE Congress on Evolutionary Computation (CEC), IEEE, 2015, pp. 2697-2704.

[19] V. Bhaskar, S. K. Gupta, A. K. Ray, Applications of multiobjective optimization in chemical engineering, Reviews in chemical engineering 16 (1) (2000) 1-54.

[20] V. Pareto, Manual of political economy, Oxford University Press, Oxford, 2014, english translation. First Edition dated of 1906.

[21] P. A. Clark, A. W. Westerberg, Optimization for design problems having more than one objective, Comput. Chem. Eng. 7 (4) (1983) 259 - 278.

[22] V. Bhaskar, S. K. Gupta, A. K. Ray, Applications of multiobjective optimization in chemical engineering, Rev. Chem. Eng. 16 (1) (2000) $1-54$.

[23] Masuduzzaman, G. P. Rangaiah, Multi-objective optimization applications in chemical engineering, in: G. P. Rangaiah (Ed.), Multi-Objective Optimization. Techniques and Applications in Chemical Engineering, World Scientific, 2009, pp. 27-59.

[24] S. Sharma, G. P. Rangaiah, Multi-objective optimization applications in chemical engineering, in: G. P. Rangaiah, A. Bonilla-Petriciolet (Eds.), Multi-Objective Optimization in Chemical Engineering: Developments and Applications, John Wiley and Sons, Ltd., 2013, pp. 35-102. 
[25] J. Höller, P. Bickert, P. Schwartz, M. von Kurnatowski, J. Kerber, N. Künzle, H.-M. Lorenz, N. Asprion, S. Blagov, M. Bortz, Parameter estimation strategies in thermodynamics, ChemEngineering 3 (2) (2019) 56.

[26] M. Ehrgott, Multicriteria optimization, Vol. 2nd ed., Springer, Berlin, 2005.

[27] R. T. Marler, J. S. Arora, Survey of multi-objective optimization methods for engineering, Structural and multidisciplinary optimization 26 (6) (2004) 369-395.

[28] G. P. Rangaiah (Ed.), Multi-Objective Optimization. Techniques and Applications in Chemical Engineering, World Scientific, 2009.

[29] G. P. Rangaiah, A. Bonilla-Petriciolet (Eds.), Multi-Objective Optimization in Chemical Engineering: Developments and Applications, John Wiley and Sons, Ltd., 2013.

[30] I. Das, J. E. Dennis, A closer look at drawbacks of minimizing weighted sums of objectives for pareto set generation in multicriteria optimization problems, Struct. Optimization 14 (1) (1997) 63-69.

[31] A. Messac, C. Puemi-Sukam, E. Melachrinoudis, Aggregate objective functions and pareto frontiers: Required relationships and practical implications, Optim. Eng. 1 (2) (2000) 171-188.

[32] A. Messac, G. J. Sundararaj, R. V. Tappeta, J. E. Renaud, Ability of objective functions to generate points on nonconvex pareto frontiers, AIAA J. 38 (6) (2000) 1084-1091. 
[33] K. Stöbener, P. Klein, S. Reiser, M. Horsch, K.-H. Küfer, H. Hasse, Multicriteria optimization of molecular force fields by pareto approach, Fluid Phase Equilib. 373 (2014) 100 - 108.

[34] S. Werth, K. Stöbener, P. Klein, K.-H. Küfer, M. Horsch, H. Hasse, Molecular modelling and simulation of the surface tension of real quadrupolar fluids, Chem. Eng. Sci. 121 (2015) 110 - 117.

[35] K. Stöbener, P. Klein, M. Horsch, K.-H. Küfer, H. Hasse, Parametrization of two-center lennard-jones plus point-quadrupole force field models by multicriteria optimization, Fluid Phase Equilib. 411 (2016) 33 - 42.

[36] E. Forte, J. Burger, K. Langenbach, H. Hasse, M. Bortz, Multi-criteria optimization for parameterization of SAFT-type equations of state for water, AIChE J. 64 (1) (2018) 226-237.

[37] J. I. S. Hernández, Multi-objective optimization in mixed integer problems: With application to the beam selection optimization problem in imrt., Ph.D. thesis, TU Kaiserslautern (2012).

[38] K. Teichert, A hyperboxing pareto approximation method applied to radiofrequency ablation treatment planning., Ph.D. thesis, TU Kaiserslautern (2013).

[39] M. Bortz, J. Burger, N. Asprion, S. Blagov, R. Böttcher, U. Nowak, A. Scheithauer, R. Welke, K.-H. Küfer, H. Hasse, Multi-criteria optimization in chemical process design and decision support by navigation on pareto sets, Comput. Chem. Eng. 60 (2014) 354 - 363. 
[40] S. Punnapala, F. M. Vargas, A. Elkamel, Parameter estimation in phase equilibria calculations using multi-objective evolutionary algorithms, in: G. P. Rangaiah, A. Bonilla-Petriciolet (Eds.), Multi-Objective Optimization in Chemical Engineering: Developments and Applications, John Wiley and Sons, Ltd., 2013, pp. 249-266.

[41] J. Burger, N. Asprion, S. Blagov, R. Böttcher, U. Nowak, M. Bortz, R. Welke, K.-H. Küfer, H. Hasse, Multi-objective optimization and decision support in process engineering implementation and application, Chem. Ing. Tech. 86 (7) (2014) 1065-1072.

[42] M. Bortz, V. Maag, J. Schwientek, R. Benfer, R. Böttcher, J. Burger, E. von Harbou, N. Asprion, K.-H. Küfer, H. Hasse, Computer Aided Chemical Engineering, 37, in: J. K. Huusom, R. Gani, K. V. Gernaey (Eds.), Decision Support by Multicriteria Optimization in Process Development: An Integrated Approach for Robust Planning and Design of Plant Experiments, Elsevier, 2015, pp. 2063-2068.

[43] J. Burger, V. Papaioannou, S. Gopinath, G. Jackson, A. Galindo, C. S. Adjiman, A hierarchical method to integrated solvent and process design of physical $\mathrm{CO}_{2}$ absorption using the SAFT- $\gamma$ Mie approach, AIChE J. 61 (10) (2015) 3249-3269.

[44] N. Asprion, R. Benfer, S. Blagov, R. Böttcher, M. Bortz, M. Berezhnyi, J. Burger, E. von Harbou, K.-H. Küfer, H. Hasse, Ines an interface between experiments and simulation to support the development of robust process designs, Chem. Ing. Tech. 87 (12) (2015) 1810-1825. 
[45] N. Asprion, S. Blagov, R. Böttcher, J. Schwientek, J. Burger, E. von Harbou, M. Bortz, Simulation and multicriteria optimization under uncertain model parameters of a cumene process, Chem. Ing. Tech. 89 (5) (2017) 665-674.

[46] M. Bortz, J. Burger, E. von Harbou, M. Klein, J. Schwientek, N. Asprion, R. Böttcher, K.-H. Küfer, H. Hasse, Efficient approach for calculating pareto boundaries under uncertainties in chemical process design, Ind. Eng. Chem. Res. 56 (44) (2017) 12672-12681.

[47] E. Forte, E. von Harbou, J. Burger, N. Asprion, M. Bortz, Optimal design of laboratory and pilot-plant experiments using multiobjective optimization, Chem. Ing. Tech. 89 (5) (2017) 645-654.

[48] A. Pascoletti, P. Serafini, Scalarizing vector optimization problems, J. Optimiz. Theory App. 42 (4) (1984) 499-524.

[49] J. M. Prausnitz, R. N. Lichtenthaler, E. G. de Azevedo, Molecular Thermodynamics of Fluid-Phase Equilibria, Prentice Hall, 1999.

[50] Aspen plus., http://www.aspentech.com/ (2014).

[51] T.-H. Cho, K. Ochi, K. Kojima, Isobaric vapor-liquid equilibria for binary systems with limited miscibility, water-n-amyl alcohol and waterisoamyl alcohol, Kagaku Kogaku Ronbun. 10 (2) (1984) 181-183.

[52] M. Góral, B. Wiśniewska-Goclowska, A. Maczyński, Recommended liquidliquid equilibrium data. part 4. 1-alkanolwater systems, J. Phys. Chem. Ref. Data 35 (3) (2006) 1391-1414. 
[53] J. Wang, Z. Bao, Investigation on vaporliquid equilibrium for 2-propanol + 1-butanol + 1-pentanol at 101.3 kpa, Fluid Phase Equilib. 341 (2013) $30-34$.

[54] Y.-Q. Nan, Y. J. Hou, Q.-S. Yu, Measurement of excess molar enthalpies of some alkan-1-ol/iso-propanol binary systems, Huaxue-Xuebao 56 (1998) 105-108.

[55] V. Udovenko, T. F. Manzanko, Vapour pressure in the system isopropyl alcohol-benezene-water, Russ. J. Phys. Chem. 41 (2) (1967) 197.

[56] M. Grigiante, P. Stringari, G. Scalabrin, E. C. Ihmels, K. Fischer, J. Gmehling, (vapour+ liquid+ liquid) equilibria and excess molar enthalpies of binary and ternary mixtures of isopropanol, water, and propylene, J. Chem. Thermodyn. 40 (4) (2008) 537-548.

[57] A. A. Sayar, Liquid-liquid equilibria of some water +2 -propanol + solvent ternaries, J. Chem. Eng. Data 36 (1) (1991) 61-65.

[58] K. Yokoyama, H. Matsuda, K. Kiruhara, K. Tochiga, Measurement and correlation of liquid-liquid equilibria of alcohol + water + alcohol systems and alcohol + water + alkane systems, J. Chem. Eng. Japan 38 (2012) 69-75. 